\title{
The Genetic Architecture of the Clustering of Cardiometabolic Risk Factors: A Study of 8- to 17-Year-Old Chinese Twins
}

\author{
Ji, Fuling
}

2020-10

Ji , F, Ning, F, Duan , H, Dong , L, Yang, F, Liu , Z, Song , X, Zhang, D, Wang , S , Pang , Z , Kaprio , J \& Silventoinen , K 2020 , ' The Genetic Architecture of the Clustering of Cardiometabolic Risk Factors: A Study of 8- to 17-Year-Old Chinese Twins ' , Twin Research and Human Genetics , vol. 23 , no. 5 , 1832427420000729 , pp. 283-291 . https://doi.org/10.1017/thg.202

http://hdl.handle.net/10138/328334

https://doi.org/10.1017/thg.2020.72

unspecified

acceptedVersion

Downloaded from Helda, University of Helsinki institutional repository.

This is an electronic reprint of the original article.

This reprint may differ from the original in pagination and typographic detail.

Please cite the original version. 
The genetic architecture of the clustering of cardio-metabolic risk factors: a study of 8-17 years old Chinese twins

Fuling Ji, $\mathrm{PhD}^{1,2}$, Feng Ning, $\mathrm{PhD}^{1,2,3}$, Haiping Duan, $\mathrm{PhD}^{1,2,3}$, Liyan Dong ${ }^{1,2}$, Feng Yang ${ }^{1,2}$, Zhisheng Liu ${ }^{1,2}$, Xuyan Song ${ }^{1,2}$, Dongfeng Zhang ${ }^{3}$, Shaojie Wang ${ }^{1,2,3}$, Zengchang Pang ${ }^{1,2}$, Jaakko Kaprio, $\mathrm{MD}, \mathrm{PhD}^{4,5}$, and Karri Silventoinen, $\mathrm{PhD}^{6}$

${ }^{1}$ Qingdao Center for Disease Control and Prevention, Qingdao, China

${ }^{2}$ Qingdao Institute of Preventive Medicine, Qingdao, China

${ }^{3}$ Department of Public Health, Qingdao University Medical College, Qingdao, China

${ }^{4}$ Department of Public Health, University of Helsinki, Helsinki, Finland

${ }^{5}$ Institute for Molecular Medicine FIMM, Helsinki, Finland

${ }^{6}$ Department of Social Research, University of Helsinki, Helsinki, Finland

Running title: Genetic architecture of cardio-metabolic factors

Contact address:

Fuling Ji

Qingdao Center for Disease Control and Prevention

No 175. Shandong Road, 266033, Qingdao, China

tel: +8613687620653

fax:+86 53285658785

Email: jifuling888@sina.com 


\section{Abstract:}

Background: We explored the genetic architecture of metabolic risk factors of

cardiovascular diseases and their clustering in Chinese boys and girls.

Methods: Seven metabolic traits (body mass index [BMI], waist circumference [WC],

systolic blood pressure [SBP], diastolic blood pressure [DBP], total cholesterol [TC],

triglyceride [TG] and uric acid [UA]) were measured in a sample of 1016 twins between 8

and 17 years of age, recruited from Qingdao Twin Registry. Cholesky, independent pathway

and common pathway models were used to identify the latent genetic structure behind the

clustering of these metabolic traits.

Results: The genetic architecture of these metabolic traits was largely similar in boys and

girls. The highest heritability was found for BMI $\left(\mathrm{a}^{2}=0.63\right)$ in boys and TC $\left(\mathrm{a}^{2}=0.69\right)$ in girls.

Three heritable factors, adiposity (BMI and WC), blood pressure (SBP and DBP) and

metabolite actor (TC, TG and UA) which formed one higher-order latent phenotype, were

identified. Latent genetic, common environmental unique environmental factors indirectly

impacted the three factors through one single latent factor.

Conclusions: Our results suggest that there is one latent factor influencing several metabolic 
traits, which are known risk factors of cardiovascular diseases, in young Chinese twins.

Latent genetic, common environmental and unique environmental factors indirectly imposed on them. These results inform strategies for gene pleiotropic discovery and intervening of CVD risk factors during childhood and adolescence.

Keywords: genetics; cardiovascular disase; children; twin study; Chinese 


\section{Introduction}

Cardiovascular diseases (CVD) are the most important causes of death worldwide and

have received increasing attention, not only in high income but also in many middle-income

countries, due to their rising prevalence(Tefera et al. 2017; Raji, Mabayoje, and Bello 2015).

Abdominal obesity, hypertension, hyperlipidemia, glucose intolerance and uric acid,

reflecting the cardiometabolic risk profile, are well known metabolic risk factors for CVD.

These factors develop early in life and track from childhood through adolescence and

adulthood(Graves et al. 2014; Umer et al. 2017). Moreover, they show mutual correlations,

and this clustering has found to be stable from childhood into adulthood (Umer et al.

2017;Díez-Fernández et al. 2015;Andersen et al. 2003). Therefore, early treatment of

adiposity in childhood and adolescence is recommended as the first-line approach to reducing

CVD risk. Hotchkiss et al(Hotchkiss et al. 2014) reported that from the $43 \%$ decline in

coronary heart disease mortality rates between 2000 and 2010, 39\% was attributed to

improvement in these risk factors. Meta-analyses of pediatric lifestyle intervention studies

have also revealed that dietary modification and increased physical activity reduce weight

and improve CVD risk factors such as dyslipidemia and hypertension, and interventions that 
improve one condition are likely to ameliorate the others. However, the mechanisms linking these conditions are not fully understood. Whether these associations are the consequences of a shared genetic factor or environmental factor underlying these CVD risk factors remains controversial.

Epidemiologic studies have found that men have a higher risk of cardiovascular mortality compared to women and have a higher risk of developing CVD at earlier onset(Leutner et al. 2016). Previous twin studies on common CVD risk factors have also established that genetic and environmental factors contributing to them often differ by sex, age and ethnicity (Liao et al. 2017;Song, Lee, and Sung 2015;Li et al. 2016;Tanaka et al. 2015;Silventoinen, Jelenkovic, et al. 2017;Poveda et al. 2017). For example, a review of twin and family studies reported that heritability estimates range from 0.25 to 0.70 for body mass index (BMI); from 0.25 to 0.60 for triglycerides (TG); from 0.50 to 0.60 for total cholesterol (TC); from 0.20 to 0.70 for resting systolic blood pressure (SBP); and from 0.10 to 0.50 for diastolic blood pressure (DBP)(Rankinen et al. 2015). Most studies reported heritability estimates of about 0.4 for uric acid (UA)(Kleber et al. 2015). Moreover, latent or unidentified genes and DNA sequence variants exerting pleiotropic influences on multiple 
CVD risk factors are found by several twin and family studies. Furthermore, recent research in male adult twins suggested that some CVD risk factors, such as adiposity, lipids and insulin resistance shared one latent genetic factor by using multivariate biometrical modeling analyses(Panizzon et al. 2015). However, a limitation is that most of the studies have been conducted in Caucasian populations following Westernized life-style leaving it open whether the results can be generalized to other populations.

We have previously reported, using data from the Chinese Qingdao Twin Study (QTR) of children and adolescent, that genetic and environment contributed to eight CVD risk factors, including BMI, waist circumference (WC), waist-to-hip ratio (WHR), SBP, DBP, TC, TG and UA and there were different heritability for those CVD risk factors by sex(Ji et al. 2014). There has been recent interest in the use of multivariate modeling approach to explore the genetic architecture underlying these CVD metabolic risk factors and to clarify whether there are age, sex, and ethnic differences in genetic pleiotropy on multiple CVD risk factors. To our knowledge, this issue has not been addressed in the Chinese population. Given the above, the aims of the present study were to:

1. Quantify genetic and environmental influences on seven CVD metabolic risk factors 
(BMI, WC, SBP, DBP, TC, TG and UA) in boys and girl aged 8-17 years.

2. Investigate the common metabolic pathophysiological underlying the clustering of CVD metabolic risk factors in boys and girls aged 8-17 years.

3. Examine whether there are different combinations of these CVD metabolic risk factors sharing genetic or environmental pathways in boys and girls aged 8-17 years.

\section{Methods}

\section{Participants}

The Qingdao Twin Study (QTR) was initiated in 1998 as part of the National Twin

Study in China which was described in detail elsewhere(Duan et al. 2013). All participants

aged 8-17 years consisting of monozygotic (MZ) and dizygotic (DZ) twins were screened

during May to August in 2006. The participants were reimbursed for travel expenses for their participation in the project. Written informed consent were obtained from the parents and verbal consent from their twin children. A questionnaire, anthropometric measurements and a fasting blood sample collection were included in the survey which is described elsewhere(Ji et al. 2014). The protocols were approved by the Institutional Review Board at Qingdao Center for Disease Control and Prevention. The current analyses are based on the 1016 
participants who were free of major medical conditions and had complete data for the used

variables.

\section{Metabolic trait measures}

BMI was calculated as body weight divided by squared body-height $\left(\mathrm{kg} / \mathrm{m}^{2}\right)$, where body

weight and height were measured in lightweight clothes and without shoes. Body weight was

rounded to the nearest $0.1 \mathrm{~kg}$ and height to the nearest centimeter. WC was measured in the

midpoint between the rib cage and the iliac crest and rounded to the nearest 0.1 centimeter.

SBP and DBP were measured in the right arm by a standard procedure using mercurial table

stand model sphygmomanometer. Three measurements were taken and the average of them

was used in the analysis. Biomedical measurements (TC, TG and UA) assay methods and

zygosity examination have been reported elsewhere(Ji et al. 2014).

\section{Statistical analysis}

Descriptive statistics were calculated using R statistical software. To obtain normal or near-normal distributions, BMI and TG were log transformed and then multiplied by 100 to increase the variance, which facilitated the model-fitting. The outliers for $\mathrm{WC}>40 \mathrm{~cm}$, $\mathrm{TC}>6.5 \mathrm{mmol} / \mathrm{L}, \mathrm{TG}>2.8 \mathrm{mmol} / \mathrm{L}, \mathrm{UA}>600 \mu \mathrm{mol} / \mathrm{L}, \mathrm{SBP}>140 \mathrm{mmHg}$ and $\mathrm{DBP}>100 \mathrm{mmHg}$ 
were removed.

Genetic analyses were performed using OpenMx(Boker et al. 2011) package of $R$

software. OpenMx estimates parameters using maximum likelihood method and computes

goodness-of-fit statistics based on minus twice the natural logarithm of the likelihood $(-2 \ln L)$.

Likelihood ratio tests (LRT) are used to test hypotheses, as a better balance between

goodness-of-fit and parsimony, the Akaike's Information Criterion (AIC) also served as an

indicator of model fit, lowest values imply the best model.

We started the multivariate modeling by fitting Cholesky decomposition which allows

partitioning the phenotypic covariance into additive genetic (A), common environment (C)

and unique environment (E) components and thus estimating genetic and environmental

correlations between pairs of traits. The Cholesky decomposition provides the full saturated

solution without making assumptions on the underlying genetic architecture. Thus, it can

supply a base model for comparsion with more parsimonious models. In a trivariate Cholesky

decomposition, we selected three phenotypic traits included obesity-related traits (BMI and

WC), blood-pressure traits (SBP and DBP), metabolites (TC, TG and UA), respectively. In

order to determine the genetic and environmental factor architecture, a series of multi-level 
independent and common pathways models were fitted to the data in boys and girls seperately. Measurement error is largely restricted to the first level of variables in these models.

We first fit a single-factor common pathway model to estimate the genetic and envrionmental covariance among latent phenotype. Then, we fit a three-correlated-factor common pathway model to estimate the phenotypic, genetic and environmental covariance among latent phenotype. The higher-order common pathway model (Figure 1a) assumes that the covariance among the three factors is accounted for by a single, higher-order latent phenotype and that the genetic and environmental covariance among the variables is accounted for by genetic and environmental influences operating through that phenotype. The higher-order independent pathways model (Figure $1 \mathrm{~b}$ ) assumes that the covariance among the three factors is accounted for by indepent, higher-order genetic and and environmental factors. The model does not require an overarching latent phenotype; it can account for the covariance via separate genetic and environmental factors that are independent of one another, and whose loadings do not have to be collinear. The model also allows for genetic and environmental factor structure to be tested separately from one another, thus remaining 
agnostic as to whether genetic and environmental influences adhere to the same covariance

structure.

Figure 1 insert here

\section{Results}

\section{Descriptive analyses}

The basic characteristics of the study cohort are presented in Table 1. The means of all

metabolic factors, except for TC and TG, were slightly higher in boys than in girls.

Significant sex differences were observed $(p<0.001)$ except for DBP.

Table 1 insert here

Table 2 shows the phenotypic correlations between the traits in boys and girls. WC-BMI

and SBP-DBP displayed the highest correlation in boys and girls, ranging from 0.77 to 0.70

and from 0.68 to 0.61 , respectively. Obesity-related measures (WC and BMI) showed

moderate correlations with SBP and UA in both sexes with the exception of with UA in girls.

However, TC showed no correlation with obesity measures and BP measures in either sex.

Weak but still significant correlations were found for TC with TG and UA ( $r=0.18$ for TG-TC

in boys and 0.17 in girls, $\mathrm{r}=0.27$ for TC-UA in girls) except for TC-UA in boys. 
Table 2 insert here

Table 3 presents the relative influences of genetic and environmental factors on the

metabolic traits based on the full ACE Cholesky decomposition. All traits were influenced by

genetic factors with the heritability estimates ranging from 0.63 for BMI to 0.22 for $\mathrm{WC}$ in

boys; the only exceptions was no significant genetic influences for DBP. In girls, the

heritability estimates vary from 0.69 for TC to 0.38 for WC with an exception of no

significant genetic influences for SBP. In addition, common environmental influences and

unique environmental influences were all significant for all measures $\left(c^{2}=0.63-0.26\right.$,

$\mathrm{e}^{2}=0.36-0.15$ in boys and $\mathrm{c}^{2}=0.52-0.19, \mathrm{e}^{2}=0.42-0.10$ in girls) in both sexes with some notable

exceptions, namely, common environmental factors showed no significant influence for TC

in boys, and for DBP, TC as well as TG in girls.

Table 3 insert here...

Table 4.1-4.3 shows the genetic and environmental correlations between each pair of

traits in based on Cholesky decomposition in boys and girls. For obesity-SBP, the common

environmental correlations were higher than the additive genetic correlations in boys

$\left(r_{C}=0.62-0.67\right.$ vs. $\left.r_{A}=0.35-0.39\right)$. The same circumstance was observed for obesity-UA in 
boys. Most of the genetic and environmental correlations were not significant in girls, with the exception of WC-BMI, SBP-DBP and TC-UA.

Table 4.1-4.3 insert here

\section{Multivariate Modeling Analyses}

Table 5 summarizes the goodness-of-fit indexes of the four multivariate models, that is,

single-indicator model, three-correlated indicator model, high-order independent pathway

ACE model and high-order common pathway ACE model in boys and girls when compared

to the full Cholesky decompositions. The high-order common pathway ACE model showed a

better fit than full ACE Cholesky decomposition and other multivariate models

( $\mathrm{BIC}=-8092.19$ in boy, $\mathrm{BIC}=-8862.57$ in girl, respectively), indicating that observed

measures covariance are modeled by three correlated indicators which referred to as obesity

(WC and BMI), blood pressure (SBP and DBP) and metabolite factor (TC, TG and UA) with

different factor loadings, and three correlated indicator are then unified into single common

latent factor. The final model is presented separately for boys (Figure 2a) and for girls (Figure

2b).

As shown in Figure 2a, for boys, factor loadings on the single common latent factor were 
higher for metabolite factor $(0.89,95 \%$ CI 0.59 to 1.00$)$ and obesity $(0.75,95 \%$ CI 0.60 to $0.92)$ than $\mathrm{BP}(0.65,95 \% \mathrm{CI} 0.49$ to 0.81$)$. Furthermore, each latent indicator was statistically significant heritable, with the estimates of 0.51 (95\% CI 0.26 to 0.76$)$ for obesity, 0.20 (95\% CI 0.04 to 0.51$)$ for BP and $0.28(95 \%$ CI 0.05 to 0.76$)$ for metabolite factor. The genetic and environmental factors account for the coherence of the three latent indicators by one common latent factor with moderate heritability $\left(\mathrm{a}^{2}=0.35,95 \%\right.$ CI 0.06 to $0.76 ; \mathrm{c}^{2}=0.63,95 \%$ CI 0.24 to $0.90 ; \mathrm{e}^{2}=0.01,95 \%$ CI 0 to 0.05$)$.

However, as shown in Figure 2b, for girls, factor loadings on the single common latent factor were lower for metabolite factor $(0.28,95 \%$ CI 0 to 0.72$)$ than obesity $(0.81,95 \%$ CI 0.43 to 1.00$)$ or blood pressure $(0.51,95 \%$ CI 0.30 to 0.87$)$. At the first higher-order common pathway level, only obesity presented statistically significant heritable with the estimates of 0.55 (95\% CI 0.32 to 0.87$)$, common environmental influences and unique environmental influences on BP $\left(\mathrm{c}^{2}=0.52,95 \%\right.$ CI 0.13 to $0.69 ; \mathrm{e}^{2}=0.36,95 \%$ CI 0.25 to 0.51$)$ and unique environmental influences on metabolic-related factor $\left(\mathrm{e}^{2}=0.30,95 \%\right.$ CI 0.11 to 0.54$)$ were statistically significant. At the second higher-order common pathway level, the effects of the genetic and environmental factors on the single common latent factor were not statistically 
significant.

Table 5 insert here.

Figure 2 insert here

\section{Discussion}

We examined the genetic architecture on seven metabolic CVD metabolic risk factors in boys and girls by a multivariate modeling approach. Among boys, we found three heritable factors behind the seven metabolic CVD risk factors: obesity (BMI and WC), blood pressure (SBP and DBP) and metabolite factors (TC, TG and UA). Obesity, blood pressure and metabolite factors were united by a higher-order common factor, which was influenced by latent genetic and environmental factors. In contrast for girls, one heritable factor was found with the estimate of 0.55 for obesity. These results help to further elucidate the physiologic mechanisms underlying the clustering of CVD risk factors and explain the strategies to prevent these CVD risk factors during childhood and adolescence. Furthermore, our findings indicate that there are differences between boys and girls in the genetic and environmental influences on these seven metabolic factors. However, it can be expected that CVD risk in adults will decrease if there are more common interventions on CVD risk factors during 
children and adolescents whatever for boys and girls.

It is well known that metabolic CVD risk factors are correlated, and obesity is usually behind the clustering of metabolic factors in children and adolescents(Silventoinen, Gouveia, et al. 2017). Our findings were not only consistent with the previous twin studies but also showed evidence for sex-specific phenotypic correlations which was much larger in boys than in girls, such as BMI relating to $\mathrm{WC}$ with the range from 0.77 to 0.70 and SBP relating to DBP with the range from 0.69 to 0.62 . In particularly, in boys, we observed significant moderate association between uric acid and other CVD risk traits except for triglycerides. In girls, uric acid only presented significant associations with total cholesterol and triglycerides Recently Luciano et al.(Luciano et al. 2017) investigated age-and sex-specific percentiles of serum uric acid in overweight/obese children and adolescent in Italy. The authors reported that higher serum uric acid was associated with an increased risk of high triglycerides and atherogenic profile in children and adolescent, and further confirmed that serum uric acid would act as marker of impaired adipogenesis which was reported already in 1998 by Matsuura et al.(Matsuura et al. 1998) Thus, we took TC, TG and UA as one unified factor when we explored the genetic background of this clustering of metabolic factors in our 
analysis to support our hypothesis which is latent genetic and environment factor indirectly impacting on three factors, namely obesity (BMI and WC), BP (SBP and DBP), and metabolites (TC, TG and UA) through one single latent factor. This factor resembles the metabolic syndrome (MetS), which is conceived as a clustering of metabolic risk factors, including visceral obesity, insulin resistance, hyperglycemia, high blood pressure, and dyslipidemia; Mets increases the risk for both cardiovascular disease and type II diabetes(Shen et al. 2003). To our knowledge, although uric acid is a CVD risk factor independent of insulin resistance(Facchini et al. 1991), the definition of MetS does not include uric acid. Our study was the first time to examine the genetic background of the clustering of metabolic traits including uric acid. Hence, our novel results would provide the opportunity to determine whether uric acid is one of components of MetS.

Compared to the similar study in adult male twins(Panizzon et al. 2015), our results demonstrated that higher-order common pathway ACE model is the final model for our data, that is, three separate factors formed by seven metabolic CVD risk variables tended to aggregate together into a single latent factor in both sex groups, especially in boys which the common latent factor with the significant genetic and environmental influences. We further 
detected the genetic and environmental influences on the common latent factor. Despite there is sex-specific difference in genetic and environmental contributions to these metabolic traits, such that female group had a higher heritability of WC, DBP, TC and UA with the range from 0.69 to 0.38 , vice versa, the heritability of BMI and TG was higher in boys than it was in girls, the hypothesized three-factor common pathway ACE model was shown to be stable across sex group.

Varying different degrees of systematic correlation between three factors stem from higher-order common pathway ACE model were observed, in boys, lipid-related factor (TC,TG and UA) and obesity appeared to the main essential features of the clustering of these CVD risk factors; while in girls, obesity and blood pressure were. However, in both sex group, the mechanisms linking obesity, blood pressure and lipid-related factor (TC,TG and UA) can both be explained by genetic, common environmental and unique environmental factor, although genetic and environmental influences did not achieve statistical significant in girls. Genome-wide association studies (GWAS) studies have revealed pleiotropic effects on CVD risk factors(MacArthur et al. 2017). Kleber et al.(Kleber et al. 2015) studied pleiotropic effects of uric acid SNPs (single-nucleotide polymorphisms) and other CVD risk 
factors, and identified that two SNPs were associated with TC(rs675209 and rs17050272), one SNP was associated with TG (rs1260326), three SNPs were associated with BMI (rs2231142, rs10480300, and rs3741414), five SNPs were associated with SBP (rs17632159, rs1165151, rs10480300, rs1171614, and rs2078267), and five SNPs were associated with DBP (rs17632159, rs1165151, rs10480300, rs1171614, and rs675209). Thus, our results will provide the support to find pleiotropic genes affecting CVD risk factors in Chinese young population. Further, past studies based on children and young adults have suggested that obesity associated.

Interestingly in our study it was shown that common environmental influences such as diet and lifestyle played an important role underlying association between these metabolic traits, especially in girls. This finding is supported by previous studies in adult populations (Recchioni et al. 2017). Ambrosini et al.(Ambrosini et al. 2013) reported that increased sugar-sweetened beverage intake is associated with CVD in young people. CVD has long been recognized as lifestyle-related disease, and physical inactivity is regarded as a major factor which is related to CVD. It suggests that we should focus on this age group because it is a critical period for physical and behavioral development. 
In summary, this study is one of few studies to examine genetic architecture on several

metabolic risk factors on CVD, especially in a non-Caucasian population. We identified three heritable factors, that is, obesity, blood pressure and metabolites (TC, TG and UA), which united one single phenotype in both sex groups, and genetic and environmental factors indirectly impact on them. These findings suggest that it would helpful to implement considerable interventions in CVD risk factor management as early as childhood and adolescent. In addition, further research investigating the impact of specific genes and environmental factors on CVD risk factors will continue to provide insight into the most successful CVD therapies. Current health recommendations need to be adjusted accordingly.

\section{Acknowledgments}

This study was supported by the EFSD/CDS/Lilly Research Fellowship 2009 and the Natural Science Foundation of Shandong Province (\# ZR2009CM111).

\section{References}

Ambrosini, Gina Leslie, Wendy Hazel Oddy, Rae Chi Huang, Trevor Anthony Mori, Lawrence Joseph Beilin, and Susan Ann Jebb. (2013). "Prospective Associations between Sugar-Sweetened Beverage Intakes and Cardiometabolic Risk Factors in Adolescents." The American Journal of Clinical Nutrition 98(2):327-34. 
Andersen, L. B., N. Wedderkopp, H. S. Hansen, A. R. Cooper, and K. Froberg. (2003). "Biological Cardiovascular Risk Factors Cluster in Danish Children and Adolescents: The European Youth Heart Study." Preventive Medicine 37(4):363-67.

Boker, Steven, Michael Neale, Hermine Maes, Michael Wilde, Michael Spiegel, Timothy Brick, ... John Fox. (2011). “OpenMx: An Open Source Extended Structural Equation Modeling Framework." Psychometrika 76(2):306-17.

Díez-Fernández, Ana, Mairena Sánchez-López, Roberto Gulías-González, Blanca Notario-Pacheco, Jorge Cañete García-Prieto, Natalia Arias-Palencia, and Vicente Martínez-Vizcaíno. (2015). "BMI as a Mediator of the Relationship between Muscular Fitness and Cardiometabolic Risk in Children: A Mediation Analysis." PloS One 10(1):e0116506.

Duan, Haiping, Feng Ning, Dongfeng Zhang, Shaojie Wang, Dong Zhang, Qihua Tan, ... Zengchang Pang.(2013). “The Qingdao Twin Registry: A Status Update.” Twin Research and Human Genetics: The Official Journal of the International Society for Twin Studies 16(1):79-85.

Facchini, F., Y. D. Chen, C. B. Hollenbeck, and G. M. Reaven. (1991). "Relationship between Resistance to Insulin-Mediated Glucose Uptake, Urinary Uric Acid Clearance, and Plasma Uric Acid Concentration.” JAMA 266(21):3008-11.

Graves, L., S. P. Garnett, C. T. Cowell, L. A. Baur, A. Ness, N. Sattar, and D. A. Lawlor. (2014). "Waist-to-Height Ratio and Cardiometabolic Risk Factors in Adolescence: Findings from a Prospective Birth Cohort." Pediatric Obesity 9(5):327-38.

Hotchkiss, Joel W., Carolyn A. Davies, Ruth Dundas, Nathaniel Hawkins, Pardeep S. Jhund, Shaun Scholes, ...Simon Capewell. (2014). "Explaining Trends in Scottish Coronary Heart Disease Mortality between 2000 and 2010 Using IMPACTSEC Model: Retrospective Analysis Using Routine Data." BMJ (Clinical Research Ed.) 348:g1088.

Ji, Fuling, Feng Ning, Haiping Duan, Jaakko Kaprio, Dongfeng Zhang, Dong Zhang, ... Karri Silventoinen. (2014). "Genetic and Environmental Influences on Cardiovascular Disease Risk Factors: A Study of Chinese Twin Children and Adolescents." Twin 
Research and Human Genetics: The Official Journal of the International Society for Twin Studies 17(2):72-79.

Kleber, Marcus E., Graciela Delgado, Tanja B. Grammer, Günther Silbernagel, Jie Huang, Bernhard K. Krämer, ... Winfried März. (2015). "Uric Acid and Cardiovascular Events: A Mendelian Randomization Study." Journal of the American Society of Nephrology : JASN 26(11):2831-38.

Leutner, Michael, Christian Göbl, Alice Wielandner, Eleonora Howorka, Marlies Prünner, Latife Bozkurt,...Alexandra Kautzky-Willer.(2016). "Cardiometabolic Risk in Hyperlipidemic Men and Women." International Journal of Endocrinology 2016:2647865.

Li, Shuxia, Kirsten Ohm Kyvik, Haiping Duan, Dongfeng Zhang, Zengchang Pang, Jacob Hjelmborg, ...Christine Dalgård. (2016). "Longitudinal Investigation into Genetics in the Conservation of Metabolic Phenotypes in Danish and Chinese Twins." PloS One 11(9):e0162805.

Liao, Chunxiao, Wenjing Gao, Weihua Cao, Jun Lv, Canqing Yu, Shengfeng Wang, ... Liming Li.(2017). “Associations Between Obesity Indicators and Blood Pressure in Chinese Adult Twins." Twin Research and Human Genetics: The Official Journal of the International Society for Twin Studies 20(1):28-35.

Luciano, Rosa, Blegina Shashaj, MariaRita Spreghini, Andrea Del Fattore, Carmela Rustico, Rita Wietrzykowska Sforza, ... Melania Manco. (2017). "Percentiles of Serum Uric Acid and Cardiometabolic Abnormalities in Obese Italian Children and Adolescents." Italian Journal of Pediatrics 43(1):3.

MacArthur, Jacqueline, Emily Bowler, Maria Cerezo, Laurent Gil, Peggy Hall, Emma Hastings, ... Helen Parkinson. (2017). “The New NHGRI-EBI Catalog of Published Genome-Wide Association Studies (GWAS Catalog)." Nucleic Acids Research 45(D1):D896-901.

Matsuura, F., S. Yamashita, T. Nakamura, M. Nishida, S. Nozaki, T. Funahashi, and Y. Matsuzawa. (1998). "Effect of Visceral Fat Accumulation on Uric Acid Metabolism in Male Obese Subjects: Visceral Fat Obesity Is Linked More Closely to Overproduction 
of Uric Acid than Subcutaneous Fat Obesity." Metabolism: Clinical and Experimental 47(8):929-33.

Panizzon, Matthew S., Richard L. Hauger, Megan Sailors, Michael J. Lyons, Kristen C. Jacobson, Ruth Murray McKenzie, ... Carol E. Franz.(2015). “A New Look at the Genetic and Environmental Coherence of Metabolic Syndrome Components." Obesity (Silver Spring, Md.) 23(12):2499-2507.

Poveda, Alaitz, Yan Chen, Anders Brändström, Elisabeth Engberg, Göran Hallmans, Ingegerd Johansson, ... Paul W. Franks. (2017). "The Heritable Basis of Gene-Environment Interactions in Cardiometabolic Traits." Diabetologia 60(3):442-52.

Raji, Yemi, Omolara Mabayoje, and Taslim Bello.(2015). "Familial Clustering of Risk Factors for Cardiovascular Disease among First-Degree Relatives of Patients with Chronic Kidney Disease in a Sub-Saharan African Population." Cardiovascular Journal of Africa 26(2 Suppl 1):S11-14.

Rankinen, Tuomo, Mark A. Sarzynski, Sujoy Ghosh, and Claude Bouchard. (2015). "Are There Genetic Paths Common to Obesity, Cardiovascular Disease Outcomes, and Cardiovascular Risk Factors?" Circulation Research 116(5):909-22.

Recchioni, Rina, Fiorella Marcheselli, Roberto Antonicelli, Emanuela Mensà, Raffaella Lazzarini, Antonio Domenico Procopio, and Fabiola Olivieri. (2017). "Epigenetic Effects of Physical Activity in Elderly Patients with Cardiovascular Disease." Experimental Gerontology 100:17-27.

Shen, Biing-Jiun, John F. Todaro, Raymond Niaura, Jeanne M. McCaffery, Jianping Zhang, Avron Spiro, and Kenneth D. Ward. (2003). “Are Metabolic Risk Factors One Unified Syndrome? Modeling the Structure of the Metabolic Syndrome X.” American Journal of Epidemiology 157(8):701-11.

Silventoinen, Karri, Élvio Gouveia, Aline Jelenkovic, José Maia, António M. Antunes, Miguel A. A. ... Duarte Freitas. (2017). "The Genetic Background of Metabolic Trait Clusters in Children and Adolescents." Metabolic Syndrome and Related Disorders 15(7):329-36. 
Silventoinen, Karri, Aline Jelenkovic, Reijo Sund, Yoshie Yokoyama, Yoon-Mi Hur, Wendy Cozen, ... Jaakko Kaprio. (2017). "Differences in Genetic and Environmental Variation in Adult BMI by Sex, Age, Time Period, and Region: An Individual-Based Pooled Analysis of 40 Twin Cohorts." The American Journal of Clinical Nutrition 106(2):457-66.

Song, Yun-Mi, Kayoung Lee, and Joohon Sung. (2015). "Changes in Weight and Cardiovascular Disease Risk Factors in Monozygotic Twins: The Healthy Twin Study." Twin Research and Human Genetics: The Official Journal of the International Society for Twin Studies 18(2):151-57.

Tanaka, Kentaro, Soshiro Ogata, Haruka Tanaka, Kayoko Omura, Chika Honda, Osaka Twin Research Group, and Kazuo Hayakawa. (2015). "The Relationship between Body Mass Index and Uric Acid: A Study on Japanese Adult Twins." Environmental Health and Preventive Medicine 20(5):347-53.

Tefera, Yonas Getaye, Tadesse Melaku Abegaz, Tamrat Befekadu Abebe, and Abebe Basazn Mekuria. (2017). "The Changing Trend of Cardiovascular Disease and Its Clinical Characteristics in Ethiopia: Hospital-Based Observational Study.” Vascular Health and Risk Management 13:143-51.

Umer, Amna, George A. Kelley, Lesley E. Cottrell, Peter Giacobbi, Kim E. Innes, and Christa L. Lilly. (2017). "Childhood Obesity and Adult Cardiovascular Disease Risk Factors: A Systematic Review with Meta-Analysis.” BMC Public Health 17(1):683. 
TABLE 1 Baseline characteristics of phenotypes in boys and girls

\begin{tabular}{|c|c|c|c|c|c|}
\hline \multirow[t]{4}{*}{ Characteristics } & \multirow{3}{*}{\multicolumn{2}{|c|}{$\begin{array}{l}\text { Boy } \\
\text { ( } n=498 \text { twins from } \\
249 \text { pairs) }\end{array}$}} & \multirow{3}{*}{$\begin{array}{l}\text { Girl } \\
\text { ( } n=518 \text { twins from } \\
259 \text { pairs) }\end{array}$} & & \multirow[b]{4}{*}{$P$ value } \\
\hline & & & & & \\
\hline & & & & & \\
\hline & $\operatorname{Mean}(S D)$ & Range & Mean(SD) & Range & \\
\hline Age(years) & $12.33(1.95)$ & 8.0 to 17.0 & $12.26(1.94)$ & 8.0 to 17.0 & $P=0.573$ \\
\hline $\mathrm{WC}(\mathrm{cm})$ & $65.91(9.83)$ & 16.70 to 105.0 & $63.1(8.64)$ & 18.0 to 95.50 & $p<0.0001$ \\
\hline $\mathrm{DBP}(\mathrm{mmHg})$ & $65.18(8.89)$ & 37.5 to 92.0 & $64.71(9.14)$ & 40.0 to 112.0 & $P=0.405$ \\
\hline $\mathrm{TC}(\mathrm{mmol} / \mathrm{L})$ & $3.70(0.64)$ & 2.04 to 9.44 & $3.80(0.61)$ & 1.73 to 6.03 & $P=0.014$ \\
\hline $\mathrm{TG}(\mathrm{mmol} / \mathrm{L})$ & $0.87(0.51)$ & 0.26 to 3.99 & $0.98(0.45)$ & 0.24 to 3.45 & $P<0.0001$ \\
\hline UA(umol/L) & $311.3(83.44)$ & 150.0 to 731.0 & 273.4(67.75) & 103.0 to 745.0 & $p<0.0001$ \\
\hline
\end{tabular}

Note: $\mathrm{SD}=$ standard deviation; $\mathrm{BMI}=$ body mass index; $\mathrm{DBP}=$ diastolic blood pressure; $\mathrm{SBP}=$ systolic blood pressure; $\mathrm{TC}=$ total cholesterol; $\mathrm{TG}=$ triglyceride; $\mathrm{UA}=$ uric acid; $\mathrm{WC}=$ waist circumference; $P=z$ test for sex difference in means. 
TABLE 2 Phenotypic correlations among the observed variables in boys and girls with 95\%CI

\begin{tabular}{|c|c|c|c|c|c|c|c|}
\hline & Girl & & & & & & \\
\hline Phenotype & WC & BMI & SBP & DBP & $\mathrm{TC}$ & TG & UA \\
\hline \multicolumn{8}{|l|}{ Boy } \\
\hline \multirow[t]{2}{*}{ WC } & & 0.70 & 0.33 & 0.17 & -0.03 & 0.19 & -0.02 \\
\hline & & $(0.63 ; 0.75)$ & $(0.22 ; 0.43)$ & $(0.06 ; 0.28)$ & $(-0.15 ; 0.09)$ & $(0.07 ; 0.30)$ & $(-0.14 ; 0.10)$ \\
\hline \multirow[t]{2}{*}{$\mathrm{BMI}$} & 0.77 & & 0.36 & 0.20 & 0.005 & 0.21 & 0.08 \\
\hline & $(0.72 ; 0.82)$ & & $(0.25 ; 0.46)$ & $(0.09 ; 0.31)$ & $(-0.11 ; 0.12)$ & $(0.09 ; 0.32)$ & $(-0.04 ; 0.19)$ \\
\hline \multirow[t]{2}{*}{ SBP } & 0.39 & 0.45 & & 0.62 & -0.05 & 0.23 & 0.02 \\
\hline & $(0.28 ; 0.49)$ & $(0.35 ; 0.55)$ & & $(0.55 ; 0.68)$ & $(-0.17 ; 0.06)$ & $(0.12 ; 0.33)$ & $(-0.10 ; 0.13)$ \\
\hline \multirow[t]{2}{*}{ DBP } & 0.27 & 0.33 & 0.69 & & -0.08 & 0.12 & -0.12 \\
\hline & $(0.15 ; 0.37)$ & $(0.22 ; 0.43)$ & $(0.63 ; 0.75)$ & & $(-0.19 ; 0.03)$ & $(0.01 ; 0.23)$ & $(-0.23 ;-0.01)$ \\
\hline \multirow[t]{2}{*}{$\mathrm{TC}$} & -0.03 & 0.02 & -0.14 & -0.06 & & 0.17 & 0.27 \\
\hline & $(-0.15 ; 0.09)$ & $(0.097 ; 0.14)$ & $(-0.26 ;-0.03)$ & $(-0.17 ; 0.06)$ & & $(0.05 ; 0.28)$ & $(0.16 ; 0.37)$ \\
\hline \multirow[t]{2}{*}{ TG } & 0.23 & 0.22 & 0.17 & 0.16 & 0.18 & & 0.16 \\
\hline & $(0.11 ; 0.34)$ & $(0.097 ; 0.33)$ & $(0.05 ; 0.29)$ & $(0.047 ; 0.27)$ & $(0.056 ; 0.28)$ & & $(0.05 ; 0.27$ \\
\hline \multirow[t]{2}{*}{ UA } & 0.31 & 0.33 & 0.34 & 0.18 & 0.01 & 0.17 & \\
\hline & $(0.20 ; 0.42)$ & $(0.21 ; 0.43)$ & $(0.23 ; 0.45)$ & $(0.06 ; 0.29)$ & $(-0.11 ; 0.13)$ & $(0.05 ; 0.28)$ & \\
\hline
\end{tabular}

Note: $\mathrm{BMI}=$ body mass index; $\mathrm{DBP}=$ diastolic blood pressure; $\mathrm{SBP}=$ systolic blood pressure; $\mathrm{TC}=$ total cholesterol; $\mathrm{TG}=$ triglyceride; $\mathrm{UA}=$ uric acid; $\mathrm{WC}=$ waist circumference. Results are derived from the multivariate ACE Cholesky model. 
TABLE 3 Genetic and environmental influences on observed variables in boys and girls with $95 \% \mathrm{Cl}$

\begin{tabular}{|c|c|c|c|c|c|c|}
\hline \multirow[b]{2}{*}{ Phenotype } & \multicolumn{2}{|c|}{ Additive genetic influences $\left(\mathrm{a}^{2}\right)$} & \multicolumn{2}{|c|}{$\begin{array}{l}\text { Common environmental influences } \\
\left(c^{2}\right)\end{array}$} & \multicolumn{2}{|c|}{$\begin{array}{l}\text { Unique environmental influences } \\
\left(\mathrm{e}^{2}\right)\end{array}$} \\
\hline & Male & female & male & female & male & female \\
\hline \multirow[t]{2}{*}{ WC } & 0.22 & 0.38 & 0.63 & 0.52 & 0.15 & 0.10 \\
\hline & $(0.08 ; 0.44)$ & $(0.22 ; 0.61)$ & $(0.41 ; 0.77)$ & $(0.29 ; 0.68)$ & $(0.11 ; 0.20)$ & $(0.07 ; 0.13)$ \\
\hline \multirow[t]{2}{*}{ BMI } & 0.63 & 0.38 & 0.28 & 0.45 & 0.08 & 0.17 \\
\hline & $(0.46 ; 0.81)$ & $(0.19 ; 0.65)$ & $(0.11 ; 0.46)$ & $(0.18 ; 0.64)$ & $(0.06 ; 0.12)$ & $(0.13 ; 0.23)$ \\
\hline \multirow[t]{2}{*}{ SBP } & 0.23 & 0.18 & 0.60 & 0.46 & 0.17 & 0.36 \\
\hline & $(0.065 ; 0.47)$ & $(0 ; 0.55)$ & $(0.36 ; 0.75)$ & $(0.098 ; 0.67)$ & $(0.13 ; 0.23)$ & $(0.28 ; 0.46)$ \\
\hline \multirow[t]{2}{*}{ DBP } & 0.09 & 0.38 & 0.56 & 0.20 & 0.35 & 0.42 \\
\hline & $(0.0073 ; 0.31)$ & $(0.07 ; 0.62)$ & $(0.34 ; 0.68$ & $(0 ; 0.47)$ & $(0.28 ; 0.44)$ & $(0.33 ; 0.54)$ \\
\hline \multirow[t]{2}{*}{ TC } & 0.54 & 0.69 & 0.10 & 0.04 & 0.36 & 0.27 \\
\hline & $(0.22 ; 0.70)$ & $(0.42 ; 0.78)$ & $(0 ; 0.38)$ & $(0 ; 0.29)$ & $(0.28 ; 0.47)$ & $(0.21 ; 0.35)$ \\
\hline \multirow[t]{2}{*}{ TG } & 0.42 & 0.33 & 0.26 & 0.40 & 0.32 & 0.28 \\
\hline & $(0.12 ; 0.69)$ & $(0.06 ; 0.62)$ & $(0.018 ; 0.53)$ & $(-0.11 ; 0.63)$ & $(0.24 ; 0.41)$ & $(0.21 ; 0.36)$ \\
\hline \multirow[t]{2}{*}{ UA } & 0.51 & 0.55 & 0.32 & 0.19 & 0.17 & 0.26 \\
\hline & $(0.27 ; 0.77)$ & $(0.22 ; 0.78)$ & $(0.064 ; 0.55)$ & $(0 ; 0.51)$ & $(0.13 ; 0.22)$ & $(0.20 ; 0.33)$ \\
\hline
\end{tabular}

Note: $\mathrm{BMI}=$ body mass index; $\mathrm{DBP}=$ diastolic blood pressure; $\mathrm{SBP}=$ systolic blood pressure; $\mathrm{TC}=$ total cholesterol; $\mathrm{TG}=$ triglyceride; $\mathrm{UA}=$ uric acid; $\mathrm{WC}=$ waist circumference. Results are derived from the multivariate ACE Cholesky model. 
TABLE 4.1 Additive Genetic Correlations of observed variable pairs in boys and girls with 95\% CI

\begin{tabular}{|c|c|c|c|c|c|c|c|}
\hline & Girl & & & & & & \\
\hline Phenotype & WC & $\mathrm{BMI}$ & SBP & DBP & $\mathrm{TC}$ & TG & UA \\
\hline \multicolumn{8}{|l|}{ Boy } \\
\hline \multirow[t]{2}{*}{ WC } & & 0.53 & 0.34 & 1.01 & 0.33 & 0.02 & -3.44 \\
\hline & & $(0.30 ; 0.85)$ & $(-0.17 ; 1.02)$ & (NS) & (NS) & (NS) & (NS) \\
\hline \multirow[t]{2}{*}{ BMI } & 0.43 & & 0.42 & 0.85 & 3.23 & 0.18 & 1.46 \\
\hline & $(0.24 ; 0.68)$ & & $(-0.08 ; 1.10)$ & (NS) & (NS) & $(-0.86 ; 1.26)$ & (NS) \\
\hline \multirow[t]{2}{*}{ SBP } & 0.35 & 0.39 & & 0.25 & 0.61 & 0.25 & -1.68 \\
\hline & $(0.01 ; 0.79)$ & $(0.09 ; 0.77)$ & & $(-0.11 ; 0.72)$ & (NS) & (NS) & (NS) \\
\hline \multirow[t]{2}{*}{ DBP } & 0.45 & 0.58 & 0.18 & & 0.61 & 0.37 & 1.06 \\
\hline & $(-0.01 ; 1.20)$ & $(0.12 ; 1.17)$ & $(-0.00 ; 0.48)$ & & (NS) & (NS) & (NS) \\
\hline \multirow[t]{2}{*}{$\mathrm{TC}$} & -3.32 & 1.60 & 1.02 & 0.07 & & 1.33 & 0.68 \\
\hline & (NS) & (NS) & (NS) & (NS) & & (NS) & $(0.24 ; 1.33)$ \\
\hline \multirow[t]{2}{*}{ TG } & 0.42 & 0.61 & -0.18 & 0.23 & 0.81 & & 0.12 \\
\hline & $(-0.39 ; 1.43)$ & $(-0.24 ; 1.55)$ & (NS) & (NS) & (NS) & & (NS) \\
\hline \multirow[t]{2}{*}{ UA } & 0.33 & 0.37 & 0.23 & 0.29 & -8.61 & -0.38 & \\
\hline & $(-0.17 ; 0.99)$ & $(-0.09 ; 0.91)$ & $(-0.26 ; 0.08)$ & (NS) & (NS) & (NS) & \\
\hline
\end{tabular}

Note: $\mathrm{BMI}=$ body mass index; $\mathrm{DBP}=$ diastolic blood pressure; $\mathrm{SBP}=$ systolic blood pressure; $\mathrm{TC}=$ total cholesterol; $\mathrm{TG}=$ triglyceride; $\mathrm{UA}=$ uric acid; $\mathrm{WC}=$ waist circumference. Results are derived from the multivariate ACE Cholesky model. 
TABLE 4.2 Common environmental correlations of observed variable pairs in boys and girls with $95 \% \mathrm{Cl}$

\begin{tabular}{|c|c|c|c|c|c|c|c|}
\hline & Girl & & & & & & \\
\hline Phenotype & WC & $\mathrm{BMI}$ & SBP & DBP & $\mathrm{TC}$ & TG & UA \\
\hline \multicolumn{8}{|l|}{ Boy } \\
\hline \multirow[t]{2}{*}{ WC } & & 0.40 & 0.61 & 0.01 & 1.43 & 0.70 & 4.12 \\
\hline & & $(0.08 ; 0.63)$ & $(-0.06 ; 1.09)$ & (NS) & (NS) & (NS) & (NS) \\
\hline \multirow[t]{2}{*}{ BMI } & 0.49 & & 0.53 & 0.16 & -6.60 & 0.58 & -0.19 \\
\hline & $(0.24 ; 0.68)$ & & $(-0.14 ; 1.00)$ & (NS) & (NS) & (NS) & (NS) \\
\hline \multirow[t]{2}{*}{ SBP } & 0.67 & 0.62 & & 0.43 & 0.50 & 0.71 & 3.82 \\
\hline & $(0.24 ; 0.98)$ & $(0.25 ; 0.91)$ & & $(-0.02 ; 0.76)$ & (NS) & $(-0.38 ; 1.70)$ & (NS) \\
\hline \multirow[t]{2}{*}{ DBP } & 0.57 & 0.46 & 0.65 & & 0.09 & 0.38 & -0.19 \\
\hline & $(-0.15 ; 1.02)$ & $(-0.10 ; 0.91)$ & $(0.36 ; 0.83)$ & & (NS) & (NS) & (NS) \\
\hline \multirow[t]{2}{*}{ TC } & 4.25 & -1.52 & 0.13 & 0.31 & & -0.63 & 0.03 \\
\hline & (NS) & (NS) & (NS) & (NS) & & (NS) & $(-0.66 ; 0.87)$ \\
\hline \multirow[t]{2}{*}{ TG } & 0.51 & 0.30 & 0.92 & 0.70 & -0.18 & & 0.56 \\
\hline & $(-0.48 ; 1.26)$ & $(-0.66 ; 1.10)$ & (NS) & (NS) & (NS) & & (NS) \\
\hline \multirow[t]{2}{*}{ UA } & 0.71 & 0.60 & 0.78 & 0.63 & 2.94 & 1.27 & \\
\hline & $(0.07 ; 1.19)$ & $(0.06 ; 1.04)$ & $(0.23 ; 1.25)$ & (NS) & (NS) & (NS) & \\
\hline
\end{tabular}

Note: $\mathrm{BMI}=$ body mass index; $\mathrm{DBP}=$ diastolic blood pressure; $\mathrm{SBP}=$ systolic blood pressure; $\mathrm{TC}=$ total cholesterol; $\mathrm{TG}=$ triglyceride; $\mathrm{UA}=$ uric acid; $\mathrm{WC}=$ waist circumference. Results are derived from the multivariate ACE Cholesky model. 
TABLE 4.3 Unique environmental correlations of observed variable pairs in boys and girls with $95 \% \mathrm{Cl}$

\begin{tabular}{|c|c|c|c|c|c|c|c|}
\hline & Girl & & & & & & \\
\hline Phenotype & WC & BMI & SBP & DBP & $\mathrm{TC}$ & TG & UA \\
\hline \multicolumn{8}{|l|}{ Boy } \\
\hline \multirow[t]{2}{*}{ WC } & & 0.00 & 0.06 & -0.02 & -0.76 & 0.29 & 0.33 \\
\hline & & $(0.04 ; 0.11)$ & $(-0.04 ; 0.16)$ & $(-0.33 ; 0.21)$ & (NS) & $(0.13 ; 0.79)$ & (NS) \\
\hline \multirow[t]{2}{*}{ BMI } & 0.08 & & 0.05 & -0.01 & 4.36 & 0.23 & -0.26 \\
\hline & $(0.05 ; 0.12)$ & & $(-0.06 ; 0.17)$ & $(-0.29 ; 0.23)$ & (NS) & $(0.07 ; 0.60)$ & (NS) \\
\hline \multirow[t]{2}{*}{ SBP } & -0.02 & -0.01 & & 0.32 & -0.11 & 0.04 & -1.14 \\
\hline & $(-0.09 ; 0.06)$ & $(-0.06 ; 0.04)$ & & $(0.21 ; 0.46)$ & (NS) & $(-0.23 ; 0.30)$ & (NS) \\
\hline \multirow[t]{2}{*}{ DBP } & -0.02 & -0.04 & 0.17 & & 0.31 & 0.25 & 0.13 \\
\hline & $(-0.19 ; 0.11)$ & $(-0.15 ; 0.04)$ & $(0.11 ; 0.25)$ & & (NS) & (NS) & (NS) \\
\hline \multirow[t]{2}{*}{$\mathrm{TC}$} & 0.07 & 0.93 & -0.15 & -0.05 & & 0.31 & 0.29 \\
\hline & (NS) & (NS) & (NS) & (NS) & & (NS) & $(0.13 ; 0.57)$ \\
\hline \multirow[t]{2}{*}{ TG } & 0.07 & 0.09 & 0.26 & 0.07 & 0.38 & & 0.32 \\
\hline & $(-0.10 ; 0.27)$ & $(-0.05 ; 0.30)$ & (NS) & $(-0.41 ; 0.52)$ & (NS) & & (NS) \\
\hline \multirow[t]{2}{*}{ UA } & -0.03 & 0.04 & -0.01 & 0.09 & 6.67 & 0.11 & \\
\hline & $(-0.14 ; 0.06)$ & $(-0.03 ; 0.11)$ & $(-0.11 ; 0.09)$ & $(-0.17 ; 0.42)$ & (NS) & $(-0.17 ; 0.55)$ & \\
\hline
\end{tabular}

Note: $\mathrm{BMI}=$ body mass index; $\mathrm{DBP}=$ diastolic blood pressure; $\mathrm{SBP}=$ systolic blood pressure; $\mathrm{TC}=$ total cholesterol; $\mathrm{TG}=$ triglyceride; $\mathrm{UA}=$ uric acid; $\mathrm{WC}=$ waist circumference. Results are derived from the multivariate ACE Cholesky model. 
TABLE 5 Multivariate model-fitting results in boys and girls

\begin{tabular}{|c|c|c|c|c|c|c|c|}
\hline Model & $-2 L L$ & df & AIC & BIC & LRT & $\Delta \mathbf{d f}$ & $\mathbf{p}$ \\
\hline \multicolumn{8}{|l|}{ Boys } \\
\hline 1.ACE Cholesky & 6106.78 & 2658 & 790.78 & -7935.97 & - & - & - \\
\hline 2.Single FactorCP & 6378.7 & 2712 & 954.7 & -7949.35 & 271.92 & 54 & $8.08 \mathrm{e}-31$ \\
\hline 3.Three Factors CP & 6183.94 & 2699 & 785.94 & -8075.43 & 77.16 & 41 & $5.40 e-04$ \\
\hline 4.Higher-order IP ACE & 6184.68 & 2699 & 786.68 & -8074.69 & 77.89 & 41 & $4.47 e-04$ \\
\hline 5.Higher-order CP ACE* & 6188.31 & 2703 & 782.31 & -8092.19 & 81.52 & 45 & 7.01e-04 \\
\hline \multicolumn{8}{|l|}{ Girls } \\
\hline 1.ACE Cholesky & 6426.54 & 2831 & 764.54 & -8697.61 & - & - & - \\
\hline 2.Single Factor CP & 6691.38 & 2885 & 921.38 & -8721.25 & 264.84 & 54 & $1.41 \mathrm{e}-29$ \\
\hline 3.ThreeFactors CP & 6493.19 & 2872 & 749.19 & -8849.99 & 66.65 & 41 & 0.01 \\
\hline 4.Higher-order IP ACE & 6493.41 & 2872 & 749.41 & -8849.78 & 66.86 & 41 & 0.01 \\
\hline 5.Higher-order CP ACE* & 6501.99 & 2876 & 749.99 & -8862.57 & 75.45 & 45 & 0.003 \\
\hline
\end{tabular}

Note : $-2 \mathrm{LL}=-2 \log$ likelihood; $\mathrm{df}=$ degrees of freedom; $\mathrm{AIC}=$ Akaike's information criterion; $\mathrm{BIC}=$ Bayesian information criterion; $\mathrm{LRT}=$ likelihood ration test $\Delta \mathrm{df}=$ change in degree of freedom; $p=$ significance of $L R T ; C P=$ common pathway model; $I P=$ independent pathway model; $\mathrm{A}=$ additive genetic influences; $\mathrm{C}=$ common environmental influences; $\mathrm{E}=$ unique environmental influences; Asterisk(*) indicates best-fitting model. Through model 2 to model 5 , the fit is tested relative to model 1 . 


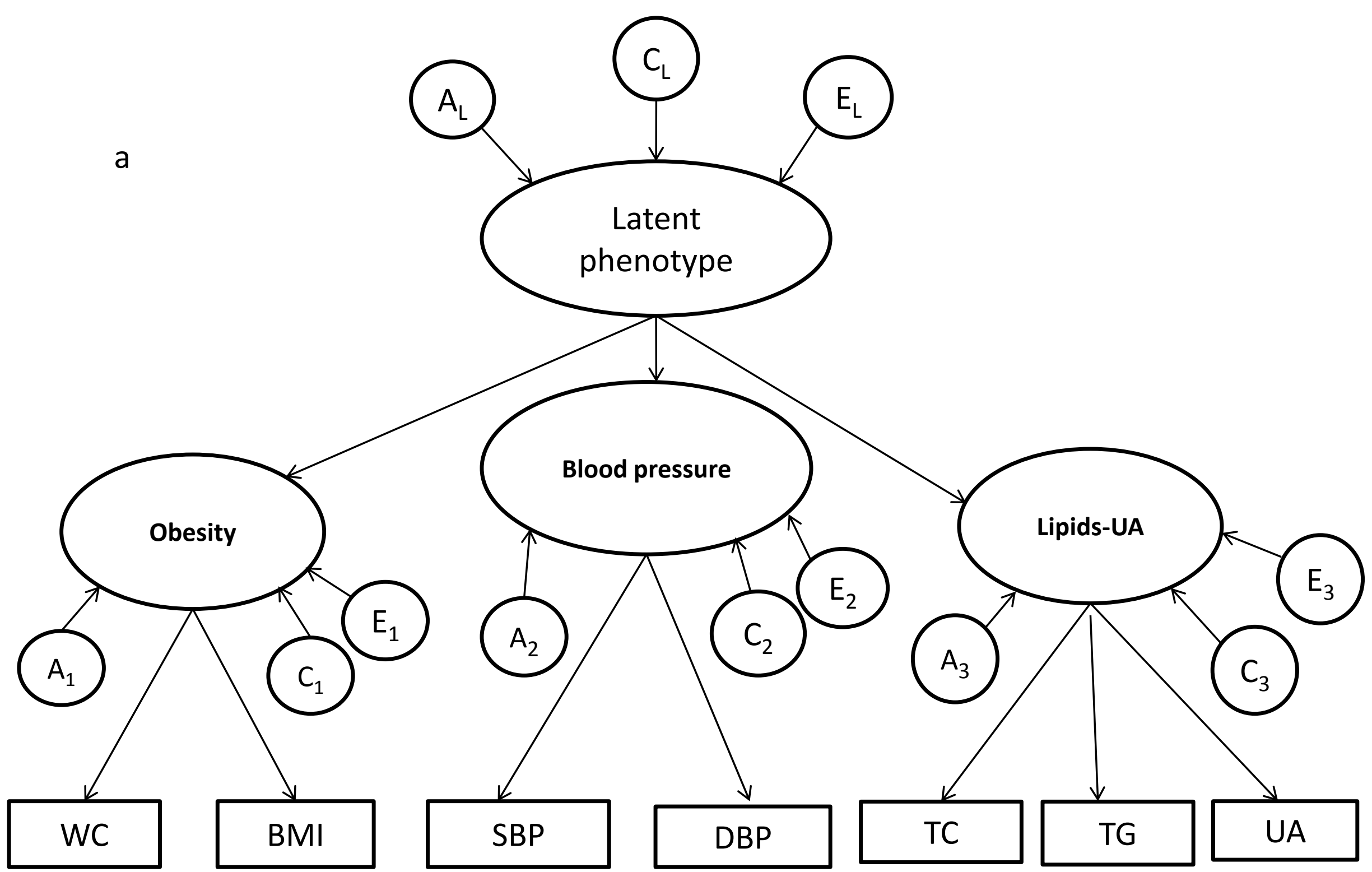

Figure 1a Higher-order common pathway models of the clustering of metabolic risk traits. Rectangles represent observed variables, ellipses represent latent factor, and circles represent latent genetic and environmental influences for the higher-order factor are designated by the subscript" $L "$; genetic and environmental influences for the three factors have numerical subscripts. 


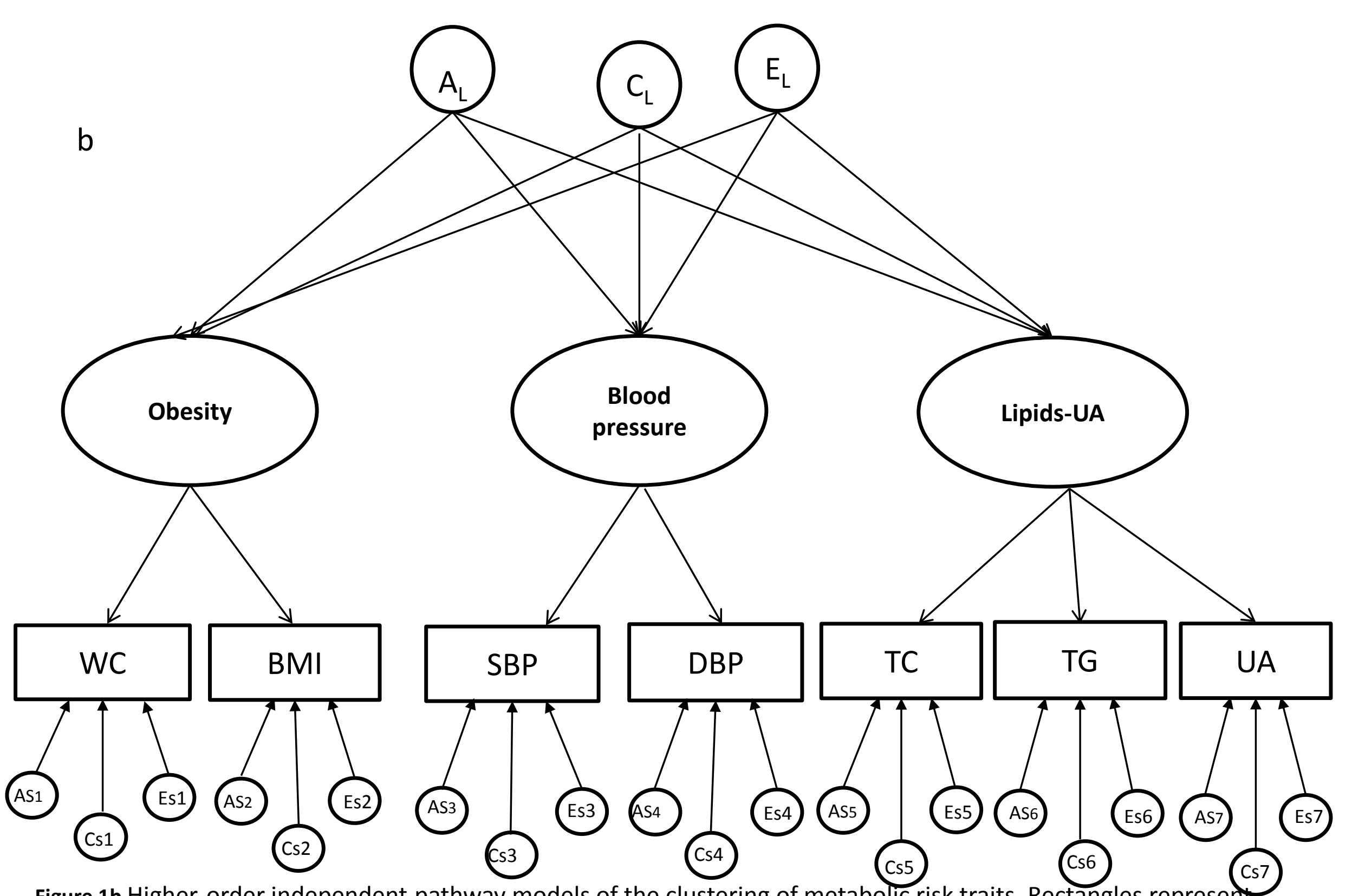

Figure 1b Higher-order independent pathway models of the clustering of metabolic risk traits. Rectangles represent observed variables, ellipses represent latent factors, and circles represent latent genetic and environmental influences for the higher-order factor are designated by the subscript" L"; genetic and environmental influences for the three factors have numerical subscripts. 

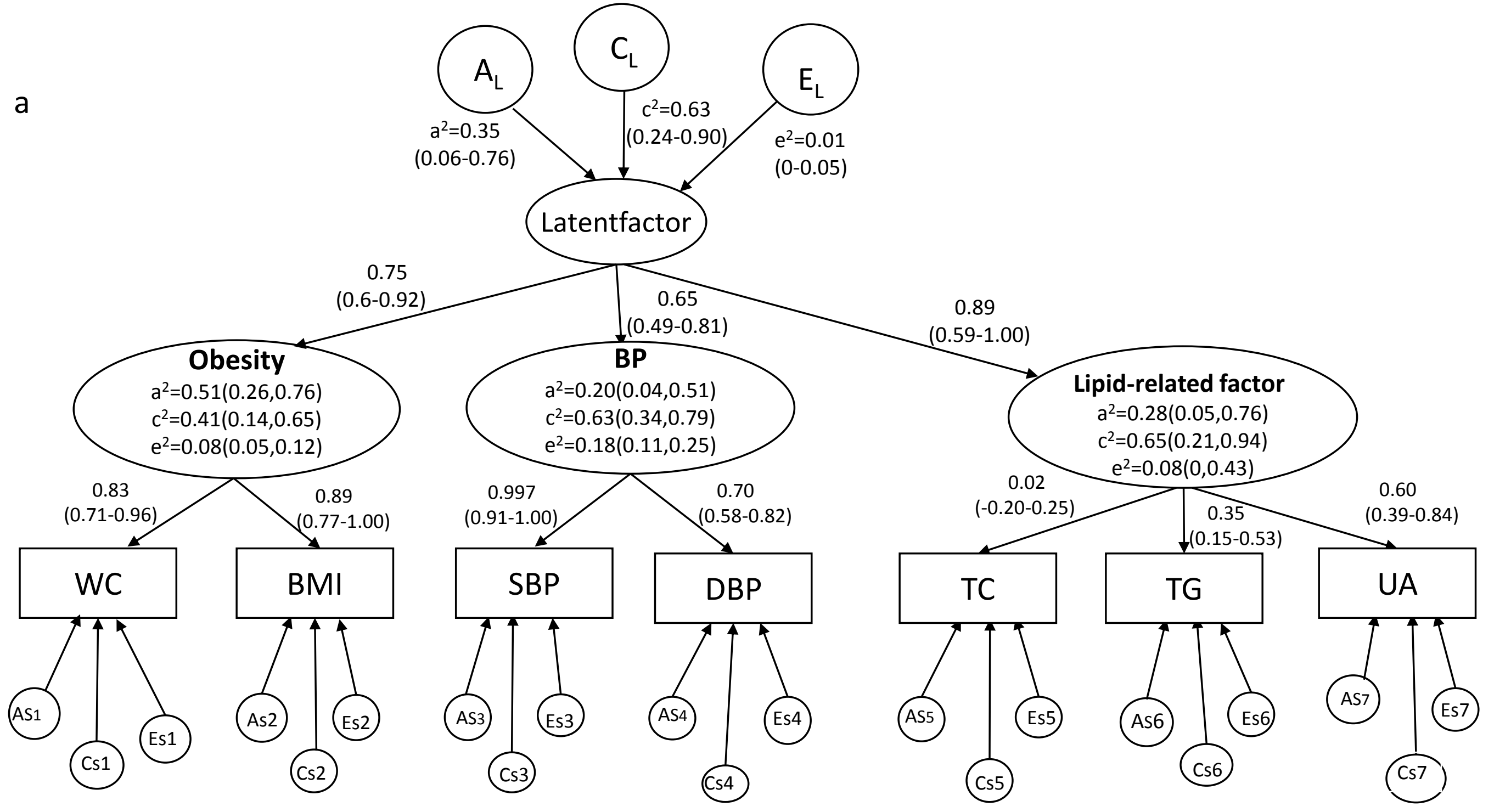

Figure 2a: Higher-order CP ACE model in boy with $95 \% \mathrm{Cl}$. As, Cs and Es are the additive genetic, common and unique environmental influences on the measured variable. 


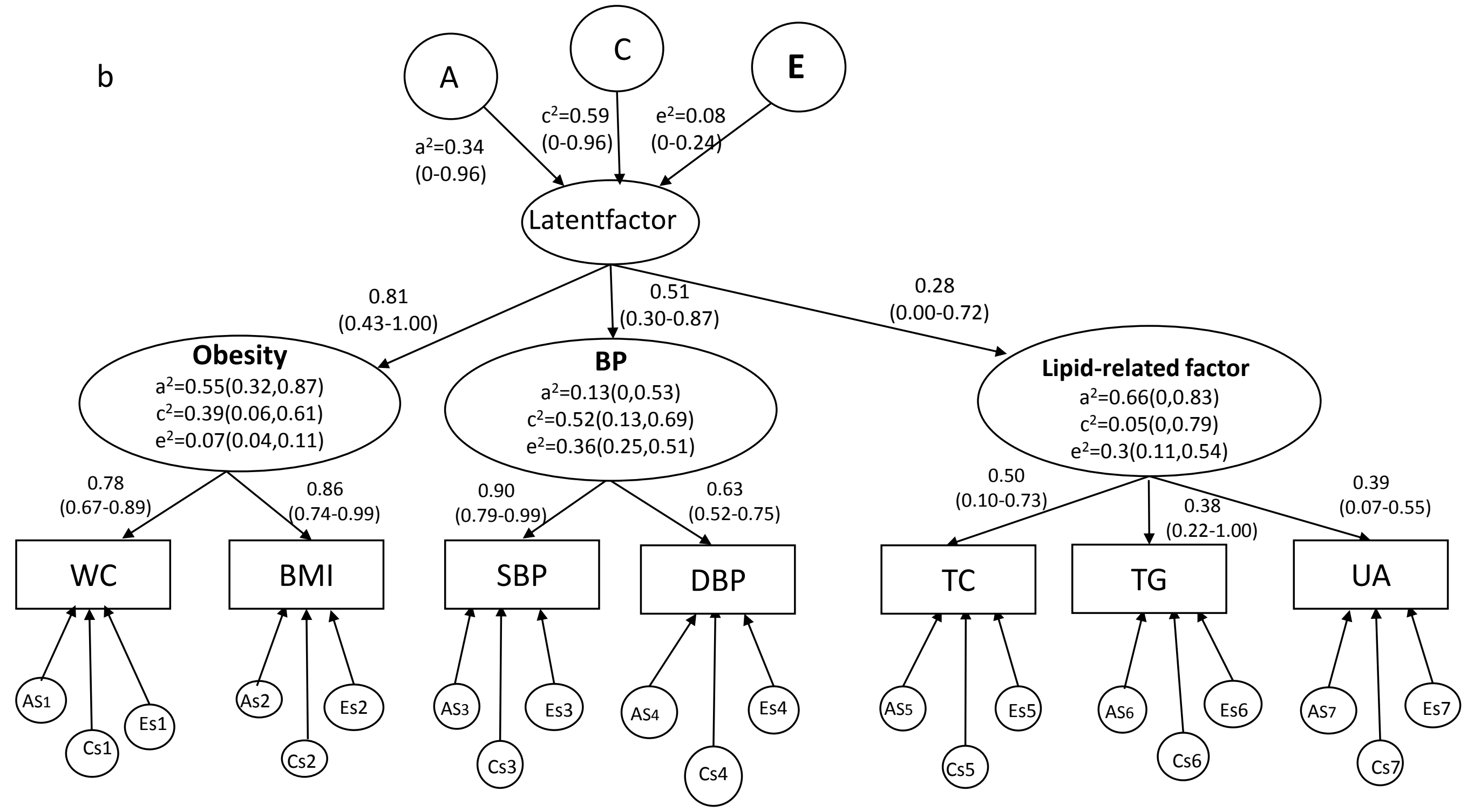

Figure 2b: Higher-order CP ACE model in girl with $95 \% \mathrm{Cl}$. As, Cs and Es are the additive genetic, common and unique environmental influences on the measured variable. 